\title{
Chronic rhinosinusitis exacerbations are differentially associated with lost productivity based on asthma status*
}

\author{
Katie M. Phillips, ${ }^{1,2}$, Regan W. Bergmark1,2, Lloyd P. Hoehle ${ }^{1,2}$, David S. \\ Caradonna ${ }^{2,3}$, Stacey T. Gray ${ }^{1,2}$, Ahmad R. Sedaghat 1,2,4, \\ ' Department of Otolaryngology, Massachusetts Eye and Ear Infirmary, Boston, MA, USA \\ 2 Department of Otolaryngology, Harvard Medical School, Boston, MA, USA \\ 'Division of Otolaryngology, Beth Israel Deaconess Medical Center, Boston, MA, USA \\ ${ }^{4}$ Department of Otolaryngology and Communications Enhancement, Boston Children's Hospital, Boston, MA, USA
}

Rhinology 56: 4, 323-329, 2018

https://doi.org/10.4193/Rhin18.033

*Received for publication:

February 16, 2018

Accepted: April 3, 2018

Background: The frequency of chronic rhinosinusitis (CRS) exacerbations is an independent predictor of quality of life. The objective of this study was to evaluate if increased CRS exacerbations predict decreased productivity.

Methods: Cross-sectional study of adult CRS patients. Number of patient-reported CRS-related antibiotic and oral corticosteroids courses and sinus infections in the past three months were used as metrics for acute exacerbations of CRS (AECRS). Productivity loss was measured by asking participants the number of lost days of work or school due to CRS in the past three months. Associations were sought between lost productivity and AECRS, controlling for clinical and demographic characteristics.

Results: 371 participants were recruited. $28.8 \%$ of study participants had comorbid asthma. The mean number of lost days of productivity due to CRS in the last three months was 1.5 for asthmatic participants and 2.4 for non-asthmatic participants. In asthmatics, CRS-related lost productivity was significantly associated with number of CRS-related antibiotics used (and oral corticosteroids used, with a trend for sinus infections. No AECRS metric was significantly associated with lost productivity in non-asthmatics. However, when focusing on non-asthmatics reporting missed days of work or school due to CRS, we found statistically significant associations between AECRS metrics and lost productivity.

Conclusions: The frequency of AECRS is associated with CRS-related lost productivity in asthmatics and in the subset of nonasthmatics with moderate CRS-related productivity losses.

Key words: acute exacerbations, asthma, chronic rhinosinusitis, lost productivity

\section{Introduction}

Chronic rhinosinusitis (CRS) is a disease characterized by chronic inflammation of the sinonasal cavity that is associated with a significant detrimental impact on quality of life (QOL) as well as decreased productivity, for example due to missed work ${ }^{(1-5)}$. The prevalence of CRS in North America and Europe is estimated to be up to $10.9 \%$, suggesting that the impact of CRS is felt by many ${ }^{(3,6)}$. A recent study found the mean work days lost per year secondary to CRS was 24.6 days per CRS patient and the annual costs associated with a single patient's productivity losses due to refractory CRS may be as high as ten thousand US dollars ${ }^{(5)}$. On a societal level, 11.5 million missed work days are attributable to CRS in the United States (U.S.) annually ${ }^{(7)}$, ranking sinusitis in the "top 10 " of most costly physical illnesses to U.S. employers in terms of productivity ${ }^{(8)}$. The indirect costs of CRS - in part due to productivity losses - across the world are estimated to be on the order of billions of U.S. dollars ${ }^{(9)}$. Given this substantial economic impact for both the individual and society as a whole, further understanding of how CRS may diminish productivity is paramount.

CRS symptom severity is known to be dominantly associated with decreased productivity due to CRS ${ }^{(5,10)}$. However, chronic symptoms and acute exacerbations of CRS (AECRS) impact patients independently and distinctly ${ }^{(11)}$. An AECRS is defined as an acute worsening of symptoms with return to baseline, often requiring a transient escalation in treatment, such as a course of 
oral antibiotics or corticosteroids ${ }^{(1)}$. AECRS can greatly impact CRS patients in many ways including increased risk of orbital or intracranial complications ${ }^{(1)}$; decreased QOL ${ }^{(11)}$; and potential for exacerbation of comorbid disease such as asthma ${ }^{(12,13)}$ which may lead to further QOL and productivity losses ${ }^{(14)}$. Despite the importance of AECRS, it is unknown how AECRS may contribute to decreased productivity. Given the variety of ways they can potentially affect CRS patients, we hypothesized that the frequency of AECRS would be associated with the number of patient-reported missed days of work or school due to CRS. Additionally, because previous work has suggested that AECRS often lead to subsequent asthma exacerbations in asthmatic CRS patients ${ }^{(12,13)}$, we hypothesized that the association between AECRS and lost productivity would be greater in asthmatic CRS patients compared to non-asthmatic CRS patients. In this study, we sought to determine whether an association exists between the frequency of AECRS and patient-reported missed days of work or school due to CRS, after stratifying patients by the presence or absence of comorbid asthma.

\section{Materials and methods}

\section{Study participants}

This study was approved by the Massachusetts Eye and Ear Infirmary Human Studies Committee. Adult patients (age 18 years or older) who met criteria for CRS based on clinical practice guidelines ${ }^{(2)}$ were recruited prospectively and provided informed consent. Exclusion criteria included comorbid diagnosis of: 1) vasculitis, 2) cystic fibrosis, 3) sarcoidosis, and/or 4) immunodeficiency. Patients with active AECRS were excluded so that all study participants' symptom severities reflected baseline symptomatology. To eliminate the possible confounding effect of recent endoscopic sinus surgery, patients who had undergone endoscopic sinus surgery within the last 6 months were also excluded.

\section{Study design and data collection}

In this cross-sectional study, all data was collected at enrollment. Demographic information was collected including age, gender and race. Tobacco use was assessed by asking participants if they were current or former smokers. Participants reported their CRS-related symptomatology as measured by the validated 22-item Sinonasal Outcomes Test (SNOT-22) survey ${ }^{(15)}$ and also reported a history of previous sinus surgery, which was confirmed by nasal endoscopy. Participants reported the number of antibiotic courses used in the past three months for CRS, number of oral corticosteroids courses used in the past three months for CRS, and the number of sinus infections experienced in the last three months, all as metrics for AECRS ${ }^{(11,16)}$. Lost productivity due to CRS was assessed by asking participants to report the number of days of work or school missed in the past three months due to $\mathrm{CRS}^{(10,17)}$. All asthmatic participants completed an Asthma Control Test (ACT) ${ }^{(18)}$ and also reported the number of oral corticosteroid courses taken for asthma in the last three months.

For each participant, the evaluating rhinologist assessed for the presence or absence of 1) asthma based on clinical consensus guidelines ${ }^{(19)}, 2$ ) aeroallergen hypersensitivity based on formal skin or serological allergy testing, ${ }^{(20)} 3$ ) aspirin sensitivity and the 4) nasal polyps on nasal endoscopy.

\section{Statistical analysis}

All analysis was performed using the statistical software package R (www.r-project.org). In order to have at least $80 \%$ power to detect an association of medium effect size (Cohen's $\mathrm{f} 2=0.15$ ) between number of missed days of work or school due to CRS and each metric of CRS exacerbations, while controlling for 8 additional covariates (specified below), 103 participants were required in the asthmatic and non-asthmatic cohorts. Patients with CRS were continuously enrolled until at least 103 participants were enrolled in each cohort. Associations between the number of missed days of work or school due to CRS as the dependent variable and the metrics of AECRS (number of CRS-related antibiotic courses, number of CRS-related oral corticosteroid courses, and number of sinus infections in the last three months) as independent variables were determined using negative binomial regression with both univariate and multivariable analyses. The multivariable regression models controlled for SNOT-22 score, age, gender, history of smoking, intranasal steroid use, aeroallergen hypersensitivity, asthma and presence of nasal polyps ${ }^{(21,22)}$. These analyses were performed for the entire cohort as well as after stratification according to the presence or absence of comorbid asthma. All correlations were performed using Pearson's method.

\section{Results}

Characteristics of study participants

A total of 371 participants with CRS were enrolled in this study. Their mean age was 52.9 (Standard Deviation [SD]:15.8); the cohort was $49.9 \%$ male and $50.1 \%$ female. The characteristics of the study participants are summarized in Table 1. Asthma was present in $28.8 \%$ of participants. $42.9 \%$ of participants had aeroallergen hypersensitivity, $4.3 \%$ had aspirin sensitivity, $46.4 \%$ had nasal polyps, $39.6 \%$ had previous sinus surgery and $46.9 \%$ reported intranasal steroid use. The mean SNOT-22 score for study participants was 34.9 (SD: 22.6). In the preceding three months, the mean number of CRS-related antibiotic courses was 0.6 (SD: 0.9), the mean number of CRS-related oral corticosteroids was 0.3 (SD: 0.8 ), and the mean number of lost work or school days due to CRS was 2.1 (SD: 8.6).

Study participants were stratified based on presence of absence of asthma (107 participants with asthma, 264 participants 
Table 1. Characteristics of study participants.

\begin{tabular}{|c|c|c|c|}
\hline & $\begin{array}{c}\text { All study } \\
\text { participants }(\mathrm{N}=\mathbf{3 7 1})\end{array}$ & $\begin{array}{c}\text { Asthmatic } \\
\text { participants }(\mathrm{N}=107)\end{array}$ & $\begin{array}{c}\text { Non-asthmatic } \\
\text { participants }(N=264)\end{array}$ \\
\hline \multicolumn{4}{|l|}{ Demographics } \\
\hline Age, mean in years, (SD) & $52.9(15.8)$ & $50.8(16.6)$ & $53.8(15.4)$ \\
\hline \multicolumn{4}{|l|}{ Gender } \\
\hline Male & $49.9 \%$ & $42.1 \%$ & $53.0 \%$ \\
\hline Female & $50.1 \%$ & $57.9 \%$ & $47.0 \%$ \\
\hline \multicolumn{4}{|l|}{ Race } \\
\hline White & $55.3 \%$ & $56.1 \%$ & $54.9 \%$ \\
\hline Black of African American & $1.1 \%$ & $1.9 \%$ & $0.8 \%$ \\
\hline Other & $3.7 \%$ & $2.9 \%$ & $3.4 \%$ \\
\hline Declined to respond & $39.9 \%$ & $39.2 \%$ & $40.9 \%$ \\
\hline Smoking & $27.8 \%$ & $29.9 \%$ & $26.9 \%$ \\
\hline \multicolumn{4}{|l|}{ Comorbidities } \\
\hline Aeroallergen hypersensitivity & $42.9 \%$ & $64.5 \%$ & $34.1 \%$ \\
\hline Asthma & $28.8 \%$ & $100.0 \%$ & $0.0 \%$ \\
\hline Aspirin sensitivity & $4.3 \%$ & $15.0 \%$ & $0.0 \%$ \\
\hline \multicolumn{4}{|l|}{ CRS characteristics } \\
\hline Nasal polyps & $46.4 \%$ & $73.8 \%$ & $35.2 \%$ \\
\hline Previous sinus surgery & $39.6 \%$ & $42.1 \%$ & $38.6 \%$ \\
\hline Intranasal steroid use & $46.9 \%$ & $67.3 \%$ & $38.6 \%$ \\
\hline SNOT-22 score, mean (SD) & $34.9(22.6)$ & $38.7(24.9)$ & $33.3(21.4)$ \\
\hline CRS-related antibiotic courses in the last 3 months, mean (SD) & $0.6(0.9)$ & $0.6(0.9)$ & $0.5(0.9)$ \\
\hline CRS-related oral corticosteroid courses in the last 3 months, mean (SD) & $0.3(0.8)$ & $0.5(0.9)$ & $0.3(0.7)$ \\
\hline Lost days of work or school in the last 3 months due to CRS, mean (SD) & $2.1(8.6)$ & $1.5(2.8)$ & $2.4(10.0)$ \\
\hline
\end{tabular}

without asthma). The mean SNOT-22 score for asthmatics was 38.7 (SD: 24.9) and the mean SNOT-22 score for participants without asthma was 33.3 (SD: 21.4). The mean number of missed days of work or school due to CRS in the last three months for asthmatics was 1.5 days (SD: 2.8 ) and for those non-asthmatic participants was 2.4 days (SD: 10.0). Neither the difference in SNOT-22 scores $(p=0.114)$ nor the difference in the number of missed days of work or school due to CRS between asthmatic and non-asthmatic study participants reached statistical significance $(p=0.142)$.

\section{CRS exacerbations are not consistently associated with lost} productivity in the general CRS patient population We first examined the relationship between missed days of work or school due to CRS and our metrics of AECRS in all study participants together, regardless of asthma status (Figure 1). In all study participants, on univariate association, we found that the number of missed days or work or school due to CRS in the past three months was not associated the number of CRS-related antibiotics courses ( $R R=1.36,95 \% \mathrm{Cl}:-0.97-1.91, \mathrm{p}=0.072)$ in the past three months. By contrast, this lost productivity was associ- ated with the number of CRS-related oral corticosteroid courses $(\mathrm{RR}=1.77,95 \% \mathrm{Cl}: 1.19-2.62, \mathrm{p}=0.005)$ and number of sinus infections ( $R R=1.59,95 \% \mathrm{Cl}: 1.04-2.43, \mathrm{p}=0.031$ ) reported in the past three months (Table 2). However, on multivariable analysis there was no statistically significant associations between missed days of work and school due to CRS and our metrics for AECRS - the number of CRS-related antibiotics courses (adjusted $\mathrm{RR}=1.43,95 \% \mathrm{Cl}$ : $1.07-1.93, \mathrm{p}=0.022$ ), number of $\mathrm{CRS}$ related oral corticosteroid courses $(R R=1.15,95 \% \mathrm{Cl}: 0.81-1.63$, $\mathrm{p}=0.421)$, or number of sinus infections ( $R R=1.40,95 \% \mathrm{Cl}$ : 0.94 $2.08, p=0.095$ ) reported in the past three months (Table 2 ).

\section{Lost days of productivity is associated with CRS exacerbati-} ons in asthmatic patients

We next stratified study participants based on presence or absence of asthma, and determined any association between the number of missed days of work or school due to CRS and AECRS (Table 2). We first examined the asthmatic cohort (Figure 2). For asthmatic participants, there were significant associations between the number of missed days of work or school due to CRS and the number of CRS-related antibiotics courses ( $R R=2.20$, 


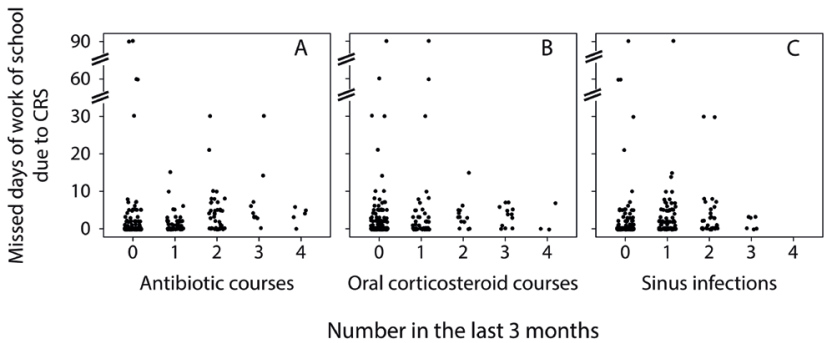

Figure 1. For all study participants, scatter plot of lost days of work or school in the last 3 months versus (A) the number of antibiotic courses taken for CRS in the last 3 months, (B) the number of oral corticosteroids courses taken for CRS in the last 3 months, and (C) the number of sinus infections in the last 3 months.

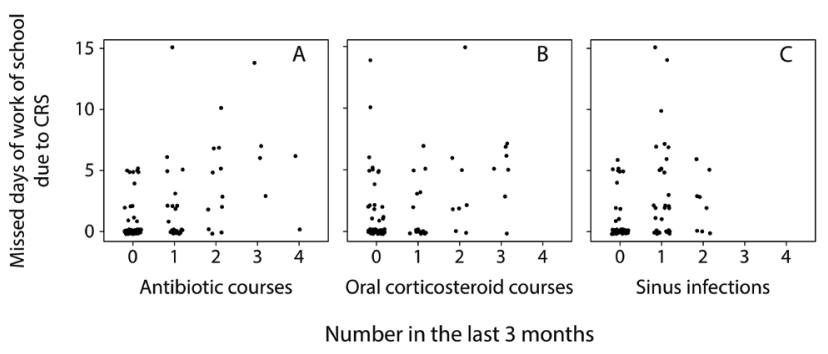

Figure 2. For asthmatic study participants, scatter plot of lost days of work or school in the last 3 months versus $(A)$ the number of antibiotic courses taken for CRS in the last 3 months, (B) the number of oral corticosteroids courses taken for CRS in the last 3 months, and (C) the number of sinus infections in the last 3 months.
$95 \% \mathrm{Cl}: 1.48-3.27, \mathrm{p}<0.001)$, number of CRS-related oral corticosteroid courses ( $R R=1.76,95 \% \mathrm{Cl}: 1.12-2.76, \mathrm{p}=0.015)$, and number of sinus infections ( $R R=3.29,95 \% \mathrm{Cl}: 1.75-6.19$, $\mathrm{p}<0.001$ ) reported in the past three months on univariate analysis. Multivariable analysis confirmed statistically significant associations between productivity loss and the number of CRSrelated antibiotics courses (adjusted RR=1.99, 95\%Cl: $1.37-2.90$, $\mathrm{p}<0.001)$ and number of CRS-related oral corticosteroid courses (adjusted RR=1.87, 95\%Cl: $1.21-2.87, \mathrm{p}=0.004$ ) reported in the past three months. The association between productivity loss and number of sinus infections (adjusted $\mathrm{RR}=1.88,95 \% \mathrm{Cl}$ : 0.98 $-3.59, p=0.057$ ) reported in the past three months did not quite reach statistical significance.

We next sought to determine to what extent CRS-related productivity losses may relate to participants' asthma severity. None of our metrics for AECRS correlated with ACT score ( $p>0.05)$. However, each metric for acute CRS exacerbations was associated with the number of asthma-related oral corticosteroid courses taken in the last three months. After controlling for either ACT score or the number of asthma-related oral corticosteroid courses, the number of CRS-related antibiotics and CRS-related oral corticosteroids courses in the past three month remained significantly associated with CRS-related lost productivity $(\mathrm{p}<$ 0.05 in all cases). Although the association between lost productivity and the number of patient-reported sinus infections did not quite reach statistical significance in the multivariable analyses above, the point estimate of the association suggested a possible association that we were simply under-powered to detect. As a result, we also checked this association after controlling for ACT and found associations with RRs ranging from 1.90 to 2.05 and $p$-values ranging from 0.032 to 0.055 . Therefore, although AECRS correlated with asthma exacerbations, the associations we found between AECRS and CRS-related lost productivity appear to be-at least to some extent—independent of asthma control.
Lost days of productivity is associated with the frequency of acute CRS exacerbations in the subset of non-asthmatics CRS patients with moderate productivity losses

We next examined the relationship between lost productivity due to CRS and AECRS for non-asthmatic study participants (Figure 3). The number of missed days of work or school due to CRS was not associated with the number of CRS-related antibiotics courses, number of CRS-related oral corticosteroid courses, or number of sinus infections reported in the past three months on multivariable analysis (Table 2). However, on visually examining the data in non-asthmatic study participants (Figure 3), we found that a small subset of non-asthmatic study participants reported extremely large productivity losses due to CRS. These outliers reduce the association between the number of missed days of work or school and frequency of acute CRS exacerbation in the prior three months, because the productivity losses greatly outstrip the number of exacerbations. In order to remove the effects of potential outliers, we excluded non-asthmatic participants reporting productivity losses greater than 1.96 times the SD ( 10 days) above the mean number ( 2.4 days) of missed days of work or school due to CRS (i.e. we excluded non-asthmatic participants reporting greater than 22 days of missed work or school due to CRS in the preceding three months). We repeated our analyses after excluding these outlying study participants and found that in non-asthmatic CRS patients with less than or equal to 22 days of CRS-related productivity losses, productivity loss was significantly associated with the number of CRS-related antibiotics courses (adjusted RR=2.03, 95\%Cl: $1.46-2.81$, $\mathrm{p}<0.001$ ) and number of sinus infections (adjusted $R R=1.57$, $95 \% \mathrm{Cl}: 1.03-2.38, \mathrm{p}=0.034)$ reported in the last three months. There was a point estimate trending towards statistical significance for number of CRS-related oral corticosteroid courses (adjusted $\mathrm{RR}=1.41,95 \% \mathrm{Cl}: 0.93-2.13, \mathrm{p}=0.108$ ) reported in the past three months. These results, showing that the number of AECRS were associated with CRS-related productivity losses in the non-asthmatic CRS patients with less than or equal to 22 days 
Table 2. Association of CRS exacerbation metrics and lost days of productivity.

\begin{tabular}{|c|c|c|c|c|}
\hline & \multicolumn{2}{|c|}{ Univariate analysis } & \multicolumn{2}{|c|}{ Multivariable analysis ${ }^{1}$} \\
\hline & $\mathbf{R R}^{2}(95 \% \mathrm{Cl})$ & $P$ value & $\mathbf{R R}^{2}(95 \% \mathrm{Cl})$ & P value \\
\hline \multicolumn{5}{|l|}{ All study participants ( $\mathrm{N}=\mathbf{3 7 1}$ ) } \\
\hline CRS-related antibiotic courses in the last 3 months, mean (SD) & $1.36(0.97-1.91)$ & 0.072 & $1.43(1.07-1.93)$ & 0.022 \\
\hline $\begin{array}{l}\text { CRS-related oral corticosteroid courses in the last } 3 \text { months, } \\
\text { mean (SD) }\end{array}$ & $1.77(1.19-2.62)$ & 0.004 & $1.15(0.81-1.63)$ & 0.421 \\
\hline Number of sinus infections in last 3 months, mean (SD) & $1.59(1.04-2.43)$ & 0.031 & $1.40(0.94-2.08)$ & 0.095 \\
\hline \multicolumn{5}{|l|}{ Asthmatic participants ( $N=107)$} \\
\hline CRS-related antibiotic courses in the last 3 months, mean (SD) & $2.20(1.48-3.27)$ & $<0.001$ & $1.99(1.37-2.90)$ & $<0.001$ \\
\hline $\begin{array}{l}\text { CRS-related oral corticosteroid courses in the last } 3 \text { months, } \\
\text { mean (SD) }\end{array}$ & $1.76(1.12-2.76)$ & 0.015 & $1.87(1.21-2.87)$ & 0.004 \\
\hline Number of sinus infections in last 3 months, mean (SD) & $3.29(1.75-6.19)$ & $<0.001$ & $1.88(0.98-3.59)$ & 0.057 \\
\hline \multicolumn{5}{|l|}{ Non-asthmatic participants ( $N=264$ ) } \\
\hline CRS-related antibiotic courses in the last 3 months, mean (SD) & $1.23(0.78-1.94)$ & 0.366 & $1.26(0.85-1.88)$ & 0.254 \\
\hline $\begin{array}{l}\text { CRS-related oral corticosteroid courses in the last } 3 \text { months, } \\
\text { mean (SD) }\end{array}$ & $2.05(1.17-3.61)$ & 0.012 & $1.06(0.66-1.71)$ & 0.808 \\
\hline Number of sinus infections in last 3 months, mean (SD) & $1.40(0.83-2.37)$ & 0.209 & $1.33(0.83-2.13)$ & 0.231 \\
\hline
\end{tabular}

${ }^{1}$ Controlling for SNOT-22 score, age, gender, smoking history, intranasal steroid use, aeroallergen hypersensitivity, asthma, polyps, and previous sinus surgery; controlling for SNOT-22 score, age, gender, smoking history, intranasal steroid use, aeroallergen hypersensitivity, polyps, and previous sinus surgery in patients stratified by asthma status. ${ }^{2}$ Relative risk.

of productivity losses, were robust to different cut-offs used for determining outliers in CRS-related lost productivity.

\section{Discussion}

Productivity is a measure of indirect costs attributed to missed days of work caused by a health condition and is one way to characterize the economic burden of a particular disease ${ }^{(17,23)}$. In today's political climate, how various chronic conditions impact productivity is especially relevant as health care resources are limited and thoughtful allocation of these resources are prioritized towards diseases causing the greatest cost burdens on society. CRS is known to be associated with a profound decrease in productivity output on both the individual and community levels ${ }^{(3)}$. Given the prevalence of $\mathrm{CRS}^{(6)}$ and its significant economic impact, understanding this association is critical to targeting how to better optimize management to prevent productivity compromise. Although acute exacerbations have recently been shown to be an important component of CRS ${ }^{(11)}$, our understanding of their impact on productivity has been limited. In this study, we found AECRS to be associated with lost productivity due to CRS independent of CRS symptom severity and that this effect was most pronounced in asthmatic patients.

Specifically, we found significant associations between lost productivity in asthmatic CRS patients and two metrics of AECRS (number of antibiotic and oral corticosteroids courses for CRS in the past three months), and a trend towards an association with our third AECRS metric (number of sinus infections in the past three months). Additionally, we found associations between two metrics for AECRS (number of antibiotic courses for CRS and number of sinus infections in the past three months) and lost productivity in the non-asthmatic CRS patients when excluding outliers reporting extremely high productivity losses, with a trend towards significance in the association between our last metric AECRS (number of oral corticosteroid courses for CRS in the last three months), and productivity loss. Interestingly, when controlling for quantifiable measures of asthma control, the association between metrics of CRS exacerbations and productivity loss remained significant in asthmatic CRS patients suggesting this relationship may be independent of asthma control. There are multiple mechanisms through AECRS may potentially reduce productivity. First, patients with an AECRS have an increased symptom burden and may simply feel too ill to work or go to school. In addition, patients with an AECRS may have to schedule an urgent clinic visit, go to the pharmacy or even get admitted to the hospital, all which may decrease daily productive output. An AECRS may potentially cause exacerbation of comorbidities, for example asthma, further adding to decreased productivity ${ }^{(11,12)}$, although our results suggest this relationship is more complex.

Given the high prevalence of comorbid asthma in CRS pa- 


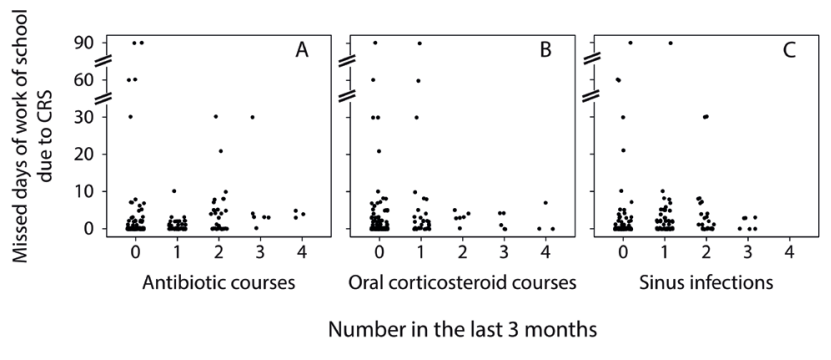

Figure 3. For non-asthmatic study participants, scatter plot of lost days of work or school in the last 3 months versus $(A)$ the number of antibiotic courses taken for CRS in the last 3 months, (B) the number of oral corticosteroids courses taken for CRS in the last 3 months, and $(C)$ the number of sinus infections in the last 3 months.

tients ${ }^{(24)}$, it is worthwhile to evaluate this subset of CRS patients. Comorbid asthma is known to be associated with other negative outcomes in patients with CRS. Previous studies have found that patients with comorbid asthma are more likely have more severe $\mathrm{CRS}^{(25)}$ or more difficult to treat CRS (26). Another study found that AECRS may precede asthma exacerbations ${ }^{(12)}$ which on their own are associated with significant, negative clinical outcomes including increased mortality, poor pulmonary function, increased healthcare expenses and decreased productivity ${ }^{(14,19)}$. One can imagine how the synergistic effect of these two diseases may lead to decreased productivity in this patient population.

Looking further into the interplay between asthma and CRS, it was interesting to find that AECRS is associated with lost productivity independent of asthma control. It is possible that AECRS may be particularly severe in asthmatic CRS patients, thereby driving lost productivity more frequently compared to non-asthmatic CRS patients. It is also possible that this finding is an artifact of how we assessed lost productivity. Since we asked participants to report the number of days of work or school missed due specifically to CRS, it is quite possible that they did not take into account the number of days missed due to asthma exacerbations that were secondary to AECRS.

Our results nevertheless highlight the importance of AECRS - independent of CRS symptom severity - as a driver of lost productivity due to CRS. The one exception to this finding was non-asthmatic CRS patients who reported extremely high productivity loss, e.g. greater than three weeks in the preceding three months. Previous work has shown mood - rather than traditional CRS disease manifestations - is a major driver of lost productivity ${ }^{(10)}$. It is possible that this dominant effect of mood, or some other unidentified factor, is even more important in the subset of patients reporting such high productivity losses due to CRS.

This study should be interpreted in the context of its limitations. There are no objective criteria for AECRS in the literature that allows direct study. Although our metrics of AECRS have been used as proxy measures for AECRS in previous studies, it is not known how well these metrics of AECRS identify every true AECRS experienced by patients. Additionally, our dependent and independent variables (lost productivity due to CRS and AECRS metrics, respectively) were based on patient recall over the preceding three months, which may introduce recall bias despite the previously described accuracy of this methodology ${ }^{(17,23)}$. Further study following these patients in a prospective manner is needed to confirm our findings. Additionally, we measured productivity as the number days of missed work or school. However, productivity loss may be experienced without missing work or school: for example, when a sick patient still is able to go to work but does not perform well, or that sick patient experiences reduced productivity in the home.

\section{Conclusion}

In conclusion, the frequency of AECRS appears to be associated with CRS-related lost productivity in asthmatics and in the subset of non-asthmatics with moderate CRS-related productivity losses. Understanding how AECRS impact productivity is the first step to better optimizing management of this CRS manifestation to prevent lost productivity due to CRS.

\section{Acknowledgement}

No funding was used to support this study. The authors gratefully acknowledge the contribution of the patients who participated in this study.

\section{Authorship contribution}

KMP, RWB, LPH, DSC, STG performed the study and wrote/revised the manuscript. ARS designed, performed the study and wrote/revised the manuscript.

\section{Conflict of interest}

The authors report no conflicts of interest.

\section{References}

1. Fokkens WJ, Lund VJ, Mullol J, et al. European position paper on rhinosinusitis and nasal polyps 2012. Rhinol Suppl. 2012;(23):298.

2. Rosenfeld RM, Piccirillo JF, Chandrasekhar SS, et al. Clinical practice guideline (update) Adult sinusitis. Otolaryngol Head Neck Surg.

\section{5;152(2 Suppl):S39.}

3. Orlandi RR, Kingdom TT, Hwang PH, et al. International consensus statement on allergy and rhinology: Rhinosinusitis. Int Forum Allergy Rhinol. 2016;6 Suppl 1:S209.

4. Hoehle LP, Phillips KM, Bergmark RW, Caradonna DS, Gray ST, Sedaghat AR Symptoms of chronic rhinosinusitis differ- entially impact general health-related quality of life. Rhinology. 2016;54(4):316-322.

5. Rudmik L, Smith TL, Schlosser RJ, Hwang $\mathrm{PH}$, Mace JC, Soler ZM. Productivity costs in patients with refractory chronic rhinosinusitis. Laryngoscope. 2014;124(9):2007-2012.

6. Hastan D, Fokkens WJ, Bachert C, et al. Chronic rhinosinusitis in Europe--an under- 
estimated disease. A GA(2)LEN study. Allergy. 2011;66(9):1216-1223.

7. Bhattacharyya N. Functional limitations and workdays lost associated with chronic rhinosinusitis and allergic rhinitis. Am J Rhino Allergy. 2012;26(2):120-122.

8. Goetzel RZ, Hawkins K, Ozminkowski RJ, Wang S. The health and productivity cost burden of the "top 10" physical and mental health conditions affecting six large U.S employers in 1999. J Occup Environ Med. 2003;45(1):5-14.

9. Smith KA, Orlandi RR, Rudmik L. Cost of adult chronic rhinosinusitis: A systematic review. Laryngoscope. 2015;125(7):15471556.

10. Campbell AP, Phillips KM, Hoehle LP, et al. Depression symptoms and lost productivity in chronic rhinosinusitis. Ann Allergy Asthma Immunol. 2017;1 18(3):286-289.

11. Phillips KM, Hoehle LP, Bergmark RW, Caradonna DS, Gray ST, Sedaghat AR. Acute exacerbations mediate quality of life impairment in chronic rhinosinusitis. J Allergy Clin Immunol Pract. 2017:5(2):422-426.

12. Ikeda K, Yokoi $H$, Kusunoki $T$, et al. Bacteriology of recurrent exacerbation of postoperative course in chronic rhinosinusitis in relation to asthma. Auris Nasus Larynx. 2011;38(4):469-473.

13. Banoub RG, Phillips KM, Hoehle LP, Caradonna DS, Gray ST, Sedaghat AR. Relationship between chronic rhinosinusitis exacerbation frequency and asthma control. Laryngoscope. 2018 May;128(5):10331038.

14. Ivanova Jl, Bergman R, Birnbaum HG, Colice GL, Silverman RA, McLaurin K. Effect of asthma exacerbations on health care costs among asthmatic patients with moderate and severe persistent asthma. J Allergy Clin
Immunol. 2012;129(5):1229-1235.

15. Hopkins C, Gillett S, Slack R, Lund VJ, Browne JP. Psychometric validity of the 22-item sinonasal outcome test. Clin Otolaryngol. 2009;34(5):447-454.

16. Yamasaki A, Hoehle LP, Phillips KM, et al. Association between systemic antibiotic and corticosteroid use for chronic rhinosinusitis and quality of life. Laryngoscope. 2018; 128(1):37-42.

17. Revicki DA, Irwin D, Reblando J, Simon GE. The accuracy of self-reported disability days. Med Care. 1994;32(4):401-404.

18. Schatz M, Sorkness CA, Li JT, et al. Asthma control test: Reliability, validity, and responsiveness in patients not previously followed by asthma specialists. J Allergy Clin Immunol. 2006;117(3):549-556.

19. EPR-3. NAEPP expert panel report 3: Guidelines for the diagnosis and treatment of asthma. Bethesda (MD): US department of health and human services; national institutes of health; national heart, lung, and blood institute, 2007.

20. Dykewicz MS, Fineman S, Skoner DP, et al. Diagnosis and management of rhinitis: Complete guidelines of the joint task force on practice parameters in allergy, asthma and immunology. american academy of allergy, asthma, and immunology. Ann Allergy Asthma Immunol. 1998;81(5 Pt 2):478-518.

21. Hoehle LP, Phillips KM, Caradonna DS, Gray ST, Sedaghat AR. A contemporary analysis of clinical and demographic factors of chronic rhinosinusitis patients and their association with disease severity. Ir J Med Sci. 2018; 187(1):215-221.

22. Campbell AP, Hoehle LP, Phillips KM, Caradonna DS, Gray ST, Sedaghat AR. Smoking: An independent risk factor for lost productivity in chronic rhinosinusitis. Laryngoscope. 2017; 127(8):1742-1745.

23. Zhang W, Bansback N, Anis AH. Measuring and valuing productivity loss due to poor health: A critical review. Soc Sci Med. 2011;72(2):185-192.

24. Rosati MG, Peters AT. Relationships among allergic rhinitis, asthma, and chronic rhinosinusitis. Am J Rhinol Allergy. 2016;30(1):4447.

25. Pearlman AN, Chandra RK, Chang D, et al. Relationships between severity of chronic rhinosinusitis and nasal polyposis, asthma, and atopy. Am J Rhinol Allergy. 2009:23(2):145-148.

26. Lopez-Chacon M, Mullol J, Pujols L. Clinical and biological markers of difficult-to-treat severe chronic rhinosinusitis. Curr Allergy Asthma Rep. 2015;15(5):19.

Ahmad R. Sedaghat, MD, PhD

Department of Otolaryngology

Massachusetts Eye and Ear Infirmary

243 Charles Street

02114 Boston, MA

USA

Tel: +1 617-573-6011

Fax: +1 617-573-6845

E-mail:

ahmad_sedaghat@meei.harvard.edu 